\title{
TAYLOR'S SERIES AND APPROXIMATION TO ANALYTIC FUNCTIONS
}

\author{
J. L. WALSH
}

Taylor's series, as the simplest expansion in terms of rational functions of an arbitrary analytic function of a complex variable, has shown itself extremely useful as a guide to other such expansions. Taylor's series itself suggests other expansions and their properties, for instance series of polynomials defined by interpolation or best approximation, and is frequently a special case or a limiting case of these expansions.

The object of this address is to indicate that Taylor's series is also useful both as a guide and as a tool in the study of still other general expansions of analytic functions, namely where each approximating function is assumed merely analytic and bounded in a given region, and where the function approximated is assumed merely analytic in a closed subregion. We shall establish certain results in detail, and later indicate some new unpublished results and some open problems.

For convenient reference we mention some properties of the Taylor development

$$
f(z)=a_{0}+a_{1} z+a_{2} z^{2}+\cdots
$$

of a function $f(z)$ analytic throughout the circle $|z|<R(>1)$ but analytic throughout no larger concentric circle. If we set

$$
S_{n}(z)=a_{0}+a_{1} z+\cdots+a_{n} z^{n},
$$

then we have

from which we find

$$
\underset{n \rightarrow \infty}{\limsup }\left|a_{n}\right|^{1 / n}=1 / R,
$$

$$
\begin{aligned}
& \limsup _{n \rightarrow \infty}\left[\max \left|f(z)-S_{n}(z)\right|, \text { for }|z|=r\right]^{1 / n}=r / R, \quad r<R \text {, } \\
& \limsup _{n \rightarrow \infty}\left[\max \left|S_{n}(z)\right|, \text { for }|z|=r\right]^{1 / n}=r / R, \quad r>R .
\end{aligned}
$$

Let us now consider the following problem, with relation to this same function $f(z)$. Let $R_{0}>R$ be fixed, and let $M>0$ be chosen; we shall later allow $M$ to become infinite. Denote by $f_{M}(z)$ the (or a)

Address of retiring vice president of the American Association for the Advancement of Science, delivered at St. Louis, March 29, 1946; received by the editors May 7, 1946. 
function analytic and of modulus not greater than $M$ in the region $|z|<R_{0}$, such that

$$
m_{M}=\left[\max \left|f(z)-f_{M}(z)\right|, \text { for }|z|=1\right]
$$

is least; thus $f_{M}(z)$ is a function of modulus not greater than $M$ in the region $|z|<R_{0}$, of best approximation to $f(z)$ on the circle $|z|=1$. It is an easily proved consequence of the theory of normal families of functions that at least one extremal function $f_{M}(z)$ exists. Moreover we have $m_{M}>0$, for otherwise $f_{M}(z)$ and $f(z)$ would coincide on the circle $|z|=1$ and throughout the region $|z|<R_{0}$, whereas $f_{M}(z)$ is analytic throughout that region and $f(z)$ has a singularity on the circle $|z|=R<R_{0}$. Obviously $m_{M}$ decreases or remains unchanged as $M$ increases. We wish to study the asymptotic relationship between $M$ and $m_{M}$, and shall proceed by employing the Taylor partial sums $S_{n}(z)$ as a comparison sequence for $f_{M}(z)$.

If $R_{1}<R$ is arbitrary, we have from (5)

$$
\left|S_{n}(z)\right| \leqq M_{1} R_{0}^{n} / R_{1}^{n}, \quad \text { for }|z|=R_{0},
$$

where $M_{1}$ is independent of $n$ and $z$; we likewise have from (4)

$$
\left|f(z)-S_{n}(z)\right| \leqq M_{2} / R_{1}^{n}, \quad \text { for }|z|=1,
$$

where $M_{2}$ is independent of $n$ and $z$. The function $S_{n}(z)$ is admitted to competition in the determination of the extremal function $f_{M}(z)$ if we choose (as we now do) $n$ corresponding to each $M$ so that we have

$$
M_{1} R_{0}^{n} / R_{1}^{n} \leqq M<M_{1} R_{0}^{n+1} / R_{1}^{n+1} ;
$$

these inequalities enable us to compare the set $f_{M}(z)$, depending on the continuous parameter $M$, with the sequence $S_{n}(z)$. We have by (8) and the first of inequalities (9)

$$
\begin{aligned}
m_{M} & =\left[\max \left|f(z)-f_{M}(z)\right|, \text { for }|z|=1\right] \\
& \leqq M_{2} / R_{1}^{n} \leqq M_{2} M / M_{1} R_{0}^{n} .
\end{aligned}
$$

By the second inequality of (9) we have

$$
\left(\log M-\log M_{1}\right) /\left(\log R_{0}-\log R_{1}\right)<n+1,
$$

so (10) can be written in the form

$$
m_{M} \leqq M_{3} M \exp \left[-\left(\log M-\log M_{1}\right) \log R_{0} /\left(\log R_{0}-\log R_{1}\right)\right],
$$

where $M_{3}$ is independent of $M$ and $n$, whence by allowing $M$ to become infinite and then by allowing $R_{1}$ to approach $R$ : 
(11) $\quad \limsup _{M \rightarrow \infty} m_{M}^{1 / \log M} \leqq \exp \left[(-\log R) /\left(\log R_{0}-\log R\right)\right]$.

Inequality (11) is one part of a main asymptotic result, for we shall now establish the following theorem.

THEOREM 1. Under the conditions imposed on $f(z)$, we have

$$
\limsup _{M \rightarrow \infty} m_{M}^{1 / \log M}=\exp \left[(-\log R) /\left(\log R_{0}-\log R\right)\right] .
$$

We establish the equality sign in (11) by use of Hadamard's threecircle theorem, that for an analytic function $\Phi(z)$, the function $\log [\max |\Phi(z)|$, for $|z|=r]$ is a convex function of $\log r$. It turns out that the strong inequality sign in (11) would imply the uniform convergence of a sequence of the functions $f_{M}(z)$ throughout a region $|z|<r(>R)$, which is impossible by the definition of $R$. Choose for definiteness the values $M=e^{n}, n=1,2, \cdots$, and denote the corresponding functions $f_{M}(z)$ by $F_{n}(z)$ respectively. Thus we have on the circle $|z|=R_{0}$ (using the Fatou boundary values on $|z|=R_{0}$, which necessarily exist)

$$
\left|F_{n}(z)\right| \leqq e^{n},\left|F_{n+1}(z)\right| \leqq e^{n+1},\left|F_{n}(z)-F_{n+1}(z)\right| \leqq 2 e^{n+1} .
$$

If we assume the first member of (11) less than the second member, we can write for $M$ sufficiently large and for suitably chosen $R_{2}$ $\left(R<R_{2}<R_{0}\right)$

$$
m_{M}^{1 / \log M} \leqq \exp \left[\left(-\log R_{2}\right) /\left(\log R_{0}-\log R_{2}\right)\right],
$$

whence from (6) for $M=e^{n}$ and $M=e^{n+1}$ we have on the circle $|z|=1$

$$
\left.\left|F_{n}(z)-F_{n+1}(z)\right| \leqq 2 \exp \left[-n \log R_{2}\right) /\left(\log R_{0}-\log R_{2}\right)\right] .
$$

The last inequality of (13), together with (14), yields by the threecircle theorem $\left(1 \leqq r \leqq R_{0}\right)$

$$
\begin{aligned}
& \underset{n \rightarrow \infty}{\limsup \left[\max \left|F_{n}(z)-F_{n+1}(z)\right|^{1 / n}, \text { for }|z|=r\right]} \\
& \leqq \exp \left[\frac{-\log R_{2}}{\log R_{0}-\log R_{2}} \cdot \frac{\log R_{0}-\log r}{\log R_{0}}+\frac{\log r}{\log R_{0}}\right] ;
\end{aligned}
$$

this last member is less than unity for every $r<R_{2}$, hence is less than unity for some value of $r, R<r<R_{2}$; the sequence $F_{n}(z)$ converges to $f(z)$ uniformly for $|z|<r$ and $f(z)$ is analytic for $|z|<r$, which contradicts the definition of $R$. Theorem 1 is established.

Suppose now we envisage a set of functions $g_{M}(z)$, analytic and re- 
spectively of modulus not greater than $M$ in the region $|z|<R_{0}$. We assume the functions $g_{M}(z)$ defined for every $M$. It is natural to study the asymptotic behavior of $g_{M}(z)$, considered to approach $f(z)$ on the circle $|z|=1$. We have by the definition of $m_{M}$

$$
\mu_{M}=\left[\max \left|f(z)-g_{M}(z)\right|, \text { for }|z|=1\right] \geqq m_{M},
$$

whence by (12):

THEOREM 2. If $f(z)$ and the functions $g_{M}(z)$ satisfy the conditions given, we have

$$
\limsup _{M \rightarrow \infty} \mu_{M}^{1 / \log M} \geqq \exp \left[(-\log R) /\left(\log R_{0}-\log R\right)\right] .
$$

Inequality (15) is our fundamental relation, which holds for the arbitrary functions $g_{M}(z)$, assumed merely analytic and individually bounded in the region $|z|<R_{0}$. However, there are numerous sets of functions, such as the partial sums of Taylor's series, and various sequences of extremal polynomials and more general rational functions for which the equality sign in (15) can be established.

In both Theorem 1 and Theorem 2 it is in fact not essential to allow $M$ to become continuously infinite; the conclusion is valid if we consider a sequence of values of $M$ such that the set of quotients of each value to its predecessor is bounded from unity and from infinity.

Theorems 1 and 2 suggest of themselves a broader problem, namely that of a more general geometric situation. Let the closed region $S$ be contained in the region $R$; let the function $f(z)$ be analytic on $S$ but not throughout $R$; we study approximation to $f(z)$ on $S$ by functions $f_{M}(z)$ respectively analytic and with upper bound not greater than $M$ in $R$. This broader problem might first be attacked by direct application of Theorems 1 and 2 using a conformal map. This method is sufficient in the case that each of the regions $S$ and $R$ is bounded by a circle-in every such case the boundaries can be transformed into concentric circles, namely the situation of Theorems 1 and 2, by means of a linear transformation of the complex variable; the second member of (12) and (15) is to be defined in terms of the largest region in which $f(z)$ is analytic, the region bounded by a level curve of the harmonic function $u(x, y)$ which takes on constant values on the boundary of $S$ and (different) constant values on the boundary of $R$. Also in case $S$ is bounded by a level curve of the Green's function for $R$, a suitable conformal transformation is adequate; the same comment on evaluating the second member of (12) and (15) applies. In more general geometric situations this simple method of conformal 
transformation is not adequate; more powerful tools must be employed. However, the new situations just treated suggest that the secret of treating the most general situation must include the use of a harmonic function and its level curves, the harmonic function being constant on the boundaries of $S$ and $R$.

We return to the situation of Theorem 1 . Instead of using in the proof of Theorem 1 the partial sums of the Taylor development of $f(z)$, we may use the sequence of rational functions $r_{n}(z)$ of respective degrees $n$ with poles uniformly distributed on the circle $|z|=R_{2}>R$ (the poles of $r_{n}(z)$ may be taken as the $n$th roots of $R_{2}{ }^{n}$ ), with $r_{n}(z)$ determined by interpolation to $f(z)$ in the $(n+1)$ th roots of unity. The convergence properties of the sequence $r_{n}(z)$ are of extremal type, in the sense that equation (12) is fulfilled, even as for the partial sums of Taylor's series. From these functions $r_{n}(z)$ we obtain not merely a new proof of Theorems 1 and 2, but a proof that in character extends to regions $S$ and $R$ of very general nature, say each bounded by a finite number of disjoint Jordan curves. In this general situation the analogue of rational functions with points of interpolation and poles uniformly distributed on circles is the set of rational functions with points of interpolation and poles uniformly distributed with respect to a parameter taken as the conjugate of the harmonic function $u(x, y)$, where $u(x, y)$ is harmonic in the region $R-S$, continuous in the corresponding closed region, and takes constant values on the boundary of $R$ and different constant values on the boundary of $S$; the points of interpolation can be uniformly distributed on the boundary of $S$; by use of a suitable conformal map, the boundary of $R$ can be chosen as one or more disjoint analytic Jordan curves; the functions $u(x, y)$ can be harmonically extended across these curves, and the poles can be prescribed on level curves of $u(x, y)$ exterior to $R$. These approximating functions were defined and studied in the present writer's book $^{1}$ in the Colloquium Series, and these functions are the only functions known which can be used for the present purpose. The threecircle theorem likewise is no longer sufficiently powerful for use in the new geometric situation, but a generalization again based on harmonic functions and due to Nevanlinna is adequate. We omit here the details, but merely refer to the generalizations of Theorems 1 and 2 set forth in papers by Walsh ${ }^{2}$ and Nilson and Walsh. ${ }^{3}$

Even in the situation of Theorem 1 itself there are numerous un-

${ }^{1}$ Interpolation and approximation by rational functions in the complex domain, Amer. Math. Soc. Colloquium Publications, vol. 20, New York, 1935.

2 Proc. Nat. Acad. Sci. U.S.A. vol. 24 (1938) pp. 477-486.

3 Trans. Amer. Math. Soc. vol. 55 (1944) pp. 53-67. 
solved problems. ${ }^{4}$ Is the extremal function $f_{M}(z)$ for prescribed $M$ unique? How can it be characterized? Does $\left|f_{M}(z)\right|$ take on (or approach) the value $M$ in an infinity of points of $|z|=R$ ? Does $\left|f(z)-f_{M}(z)\right|$ take on the value $m_{M}$ in an infinity of points of $|z|=1$ ? Do $f_{M}(z)$ and $m_{M}$ depend continuously on $M$ ? What is the relation of the entire problem of $f_{M}(z)$ to the companion problem of a prescribed $\epsilon=\left[\max \left|f(z)-\phi_{\epsilon}(z)\right|\right.$, for $\left.|z|=1\right]>0$, with $\phi_{\epsilon}(z)$ analytic and of smallest least upper bound $M_{\epsilon}$ in $|z|<R$ ? If the function $f_{M}(z)$ corresponds to the maximum error $m_{M}$, and if we choose $\epsilon=m_{M}$, can we conclude $M_{\epsilon}=M, \phi_{\epsilon}(z) \equiv f_{M}(z)$ ? If $f(z)$ is required to satisfy certain continuity conditions on $|z|=R$, does $f_{M}(z)$ satisfy corresponding conditions on $|z|=R_{0}$ ? What other measures can be used for approximation of $f_{M}(z)$ to $f(z)$ on $|z|=1$, and what norm can be used for $f_{M}(z)$ other than the least upper bound on $|z|=R_{0}$ ?

Merely for the sake of simplicity, we place ourselves anew in the situation of Theorem 1, where equation (12) holds with $m_{M}$ defined by (6), with $\left|f_{M}(z)\right| \leqq M$ in $|z|<R_{0}$, although $f_{M}(z)$ need no longer be the extremal function. The three-circle theorem as used in the proof of Theorem 1 yields further results on the degree of convergence of the set of functions $f_{M}(z)$ :

$$
\begin{aligned}
& \limsup _{M \rightarrow \infty}\left[\max \left|f(z)-f_{M}(z)\right|, \text { for }|z|=r\right]^{1 / \log M} \\
& =\exp \left[(\log r-\log R) /\left(\log R_{0}-\log R\right)\right], \quad 1 \leqq r<R, \\
& \limsup _{M \rightarrow \infty}\left[\max \left|f_{M}(z)\right| \text {, for }|z|=r\right]^{1 / \log M} \\
& =\exp \left[(\log r-\log R) /\left(\log R_{0}-\log R\right)\right], \quad R \leqq r \leqq R_{0} .
\end{aligned}
$$

In particular the functions $f_{M}(z)$ converge to $f(z)$ throughout the circle $|z|=R$, and converge in no region exterior to that circle but contained in the circle $|z|=R_{0}$. The asymptotic behavior of the set $f_{M}(z)$ on the circle $|z|=r, 1<r<R$, is the same as for the extremal functions analytic and bounded in $|z|<R_{0}$ defined by best approximation to $f(z)$ on any circle $|z|=r_{0} \leqq r$. Equations (16) and (17) are the precise analogues of (4) and (5) for Taylor's series, and indeed (4) and (5) are included in (16) and (17). Equations (4) and (5) are intimately connected with the properties of partial sums of Taylor's series: overconvergence, degree of convergence, and zeros, as studied by Porter, Jentzsch, Ostrowski, Szegö, Pólya, Carlson, Bourion, and others. A general theory of these topics has been recently developed by the

\section{Heins.}

${ }^{4}$ Some partial results here have quite recently been obtained by Professor M. H. 
present writer, and is as yet unpublished. This theory is based on the continued use of harmonic majorants, and applies in the situation of Theorem 1 and its generalization to further geometric situations. For instance, in the situation of Theorem 1, special subsequences of functions $f_{M}(z)$ may converge uniformly in a region containing an arc of the circle $|z|=R$; such overconvergence is not a local property on an arc of that circle but when it occurs must occur across every arc of that circle on which $f(z)$ is analytic. Whenever overconvergence takes place for a particular sequence of the functions $f_{M}(z)$, the first members of both (16) for $r \neq 1$ and (17) for $r \neq R$ for that sequence are less than the respective second members. When overconvergence occurs, $f(z)$ can have no isolated singularity on the circle $|z|=R$. Special subsequences of the set $f_{M}(z)$ which converge with sufficient rapidity must converge throughout the largest region in $|z|<R_{0}$ which contains $|z|<1$ and within which $f(z)$ is analytic. Every point of the circle $|z|=R$ is a limit point of the zeros of the entire set $f_{M}(z)$, and these properties hold whether $f_{M}(z)$ is extremal or not, provided equation (12) is satisfied.

We turn to another kind of problem, still for the present in the situation of Theorem 1. Can Theorem 1 be sharpened if the functions $f(z)$ and $f_{M}(z)$, the latter not necessarily extremal functions, satisfy certain continuity conditions (for instance Lipschitz conditions on the function or on some derivative) on $|z|=R$ and $|z|=R_{0}$ respectively? That is to say, Theorem 1 is primarily a relation involving on the one hand regions of analyticity of $f(z)$ and of $f_{M}(z)$ and on the other hand degree of convergence of $f_{M}(z)$ to $f(z)$. What sharper results exist when we consider in addition the behavior of $f(z)$ and $f_{M}(z)$ on the boundaries of their assigned regions of analyticity? A somewhat analogous question arises in the study of approximation of arbitrary functions by polynomials. If the functions approximated are merely assumed analytic in certain regions, a satisfactory theory can be developed relating these regions of analyticity to geometric degree of convergence of approximating polynomials. If more delicate measures of convergence are used, to construct a comprehensive theory it is essential to bring into account the behavior of the functions approximated on the boundaries of their regions of analyticity; this theory is based on studies in the real domain by S. Bernstein, Jackson, Montel, and de la Vallée-Poussin, was developed mainly by Sewell, Curtiss, and Walsh, and was recently set forth in a book by Sewell. ${ }^{5}$ The theory of approximation by polynomials, although adequate for

5 Degree of approximation by polynomials in the complex domain, Princeton, 1942. 
our present problem in case the region $R$ is bounded by a level curve of Green's function for the exterior of $S$, is not alone sufficient for the broader application we have been considering. It would seem to be necessary as a preliminary to study the convergence properties on the boundaries of regions of the sequences of rational functions used in extending the geometric situation of Theorem 1 ; no such study has ever been made. Such a study, analogous to the one made for polynomials, is highly desirable, and would have also other applications than to the principal theory considered here. Taylor's series again serves as a guide and as a model; partial but apparently typical results can be obtained readily, which seem to indicate the possibility of carrying through these programs to completion.

HARVARD UNIVERSITY 